\title{
Newer treatments for fibromyalgia syndrome
}

\author{
Richard E Harris \\ Daniel J Clauw
}

Department of Anesthesiology, The University of Michigan, Ann Arbor, MI, USA
Correspondence: Richard E Harris Chronic Pain and Fatigue Research Center, 24 Frank Lloyd Wright Drive, PO Box 385, Lobby M, Ann Arbor, MI 48I06, USA

Tel + I 7349986996

Fax +I 7349986900

Email reharris@med.umich.edu

\begin{abstract}
Fibromyalgia syndrome is a common chronic pain disorder of unknown etiology. The lack of understanding of the pathophysiology of fibromyalgia has made this condition frustrating for patients and clinicians alike. The most common symptoms of this disorder are chronic widespread pain, fatigue, sleep disturbances, difficulty with memory, and morning stiffness. Emerging evidence points towards augmented pain processing within the central nervous system (CNS) as having a primary role in the pathophysiology of this disorder. Currently the two drugs that are approved by the United States Food and Drug Administration (FDA) for the management of fibromyalgia are pregabalin and duloxetine. Newer data suggests that milnacipran, a dual norepinephrine and serotonin reuptake inhibitor, may be promising for the treatment of fibromyalgia. A double-blind, placebo-controlled trial of milnacipran in 125 fibromyalgia patients showed significant improvements relative to placebo. Milnacipran given either once or twice daily at doses up to $200 \mathrm{mg}$ /day was generally well tolerated and yielded significant improvements relative to placebo on measures of pain, patient's global impression of change in their disease state, physical function, and fatigue. Future studies are needed to validate the efficacy of milnacipran in fibromyalgia.
\end{abstract}

Keywords: fibromyalgia, pain, pharmacological, treatment

\section{Fibromyalgia in the context of other central pain syndromes}

Clinical practitioners commonly see patients with pain and other somatic symptoms that they cannot adequately explain based on the degree of damage or inflammation noted in peripheral tissues. In fact, pain may be amongst the most common ailments that individuals seek medical attention for (Khan et al 2003). Typically, an evaluation is performed looking for a "cause" for the pain. If none is found, these individuals are often given a diagnostic label that merely connotes that the patient has chronic pain in a region of the body, without an underlying mechanistic cause (eg, chronic low back pain, headache, temporomandibular disorder [TMD], etc). In other cases, the label given alludes to an underlying mechanism that may or may not be responsible for the individual's pain (eg, facet syndrome).

Fibromyalgia is merely the current term for individuals with chronic widespread musculoskeletal pain, for which no alternative cause can be identified. Gastroenterologists often see the exact same patients and focus on their gastroenterological complaints, and often use the terms functional GI disorder, irritable bowel syndrome (IBS), nonulcer dyspepsia, or esophageal dysmotility to explain the patient's symptoms (Mayer and Raybould 1990). Neurologists see these patients for their headaches and/or unexplained facial pain, urologists for pelvic pain and urinary symptoms (and use labels such as interstitial cystitis, chronic prostatitis, vulvodynia, and vulvar vestibulitis), dentists for TMD, and so on. These syndromes may have a common underlying pathology. 
Until recently these unexplained pain syndromes perplexed researchers, clinicians, and patients. However, it is now clear that:

- Individuals will sometimes only have one of these "idiopathic" pain syndromes over the course of their lifetime. But more often, individuals with one of these entities, and their family members, are likely to have several of these conditions (Hudson et al 1985; Arnold et al 2004a). Many terms have been used to describe these clustering syndromes and symptoms, including functional somatic syndromes, somatization disorders, allied spectrum conditions, chronic multisymptom illnesses, medically unexplained symptoms, etc (Hudson et al 1985; Fukuda et al 1998; Barsky and Borus 1999; Wessely et al 1999).

- Women are more likely to have these disorders than men, but the sex difference is much more apparent in clinical samples than in population-based samples (Drossman et al 1993; Aaron et al 1996).

- Groups of individuals with these conditions (eg, fibromyalgia, IBS, headache, TMD, etc) display diffuse hyperalgesia (increased pain to normally painful stimuli) and/or allodynia (pain to normally nonpainful stimuli) (Maixner et al 1995; Naliboff et al 2001; Giesecke et al 2004a, 2004b; Moshiree et al 2007). This suggests that these individuals have a fundamental problem with pain or sensory processing rather than an abnormality confined to the region of the body where the person is currently experiencing pain.

- Similar types of therapies are efficacious for all of these conditions, including both pharmacological (eg, tricyclic compounds such as amitriptyline) and nonpharmacological treatments (eg, exercise, cognitive behavioral therapy). Conversely, individuals with these conditions typically do not respond to therapies that are effective when pain is due to damage or inflammation of tissues (eg, nonsteroidal anti-inflammatory drugs [NSAIDs], opioids, injections, surgical procedures).

Until perhaps a decade ago, these conditions were all on somewhat equally tenuous scientific grounds. But within a relatively short period of time, research methods such as experimental pain testing, functional imaging, and genetics have led to tremendous advances in the understanding of several of these conditions, most notably fibromyalgia, IBS, and TMD. Many in the pain field now feel that chronic pain itself is a "disease" and that many of the underlying mechanisms operative in these heretofore-considered "idiopathic" or "functional" pain syndromes may be similar no matter whether that pain is present throughout the body (eg, in fibromyalgia) or localized to the low back, the bowel, or the bladder. Because of this, the more contemporary terms used to describe conditions such as fibromyalgia, IBS, TMD, vulvodynia, and many other entities include "central pain" "neuropathic pain" (meaning that the pain is coming from the nervous system rather than the periphery and not pain due to nerve damage), or "nonnociceptive pain" (Clauw 2007; Woolf 2004).

This review of fibromyalgia focuses on our current understanding of this disorder and its treatment with pharmacologic interventions.

\section{Historical perspective}

Although the term "fibromyalgia" is relatively new, this condition has been described in the medical literature for centuries. Sir William Gowers coined the term 'fibrositis' in 1904. During the next half century, fibrositis was considered by some to be a common cause of muscular pain, by others to be a manifestation of 'tension' or 'psychogenic rheumatism', and by the rheumatology community in general to be a non-entity. The current concept of fibromyalgia was established by Smythe and Moldofsky in the mid-1970s (1977). The name change reflected the fact that there was increasing evidence that there was no-itis or inflammation in the connective tissues of individuals with this condition, but instead - algia or pain. These authors characterized the most common tender points or regions of extreme tenderness in these individuals, and reported that patients with fibromyalgia had disturbances in deep and restorative sleep, and that selective stage 4 interruptions induced the symptoms of fibromyalgia (Moldofsky et al 1975). Yunus and colleagues (1981) then reported on the major clinical manifestations of patients with fibromyalgia seen in rheumatology clinics.

The next advance in fibromyalgia was the development of the American College of Rheumatology (ACR) criteria for fibromyalgia, which were published in 1990 (Wolfe et al 1990). These classification criteria require that an individual have both a history of chronic widespread pain (CWP), and the finding of $\geq 11$ of a possible 18 tender points on examination. These ACR classification criteria were intended for research use, to standardize definitions of fibromyalgia. In this regard, the criteria have been extremely valuable. Unfortunately, many practitioners use these criteria in routine clinical practice to diagnose individual patients, and this unintended use has led to many of the current misconceptions regarding fibromyalgia, that are discussed below.

The finding of diffusely increased tenderness, as well as a lack of finding "-itis" in the muscles or other tissues of fibromyalgia patients, caused the name of this entity to be changed from fibrositis to fibromyalgia. The diffuse nature of 
the pain and tenderness also led many groups of investigators to begin to explore neural mechanisms to explain the underlying pathogenesis of these disorders (Yunus 1992; Clauw and Chrousos 1997). In fact, major advances have only occurred in understanding individual syndromes within this spectrum once investigators concluded that this was not a condition caused by peripheral damage or inflammation, and began to explore central, neural mechanisms of these diseases. Thus, the conditions we now understand best within this spectrum include fibromyalgia, IBS, and TMD.

\section{Epidemiology}

\section{Chronic widespread pain}

Epidemiological studies of the historical component of the ACR criteria for fibromyalgia have been extremely instructive. CWP is typically operationalized as pain above and below the waist, involving the left and right sides of the body, and also involving the axial skeleton. Population-based studies of CWP suggest that $5 \%-15 \%$ of the population has this symptom at any given point in time (Croft et al 1993; Wolfe et al 1995; Cöster et al 2007). Chronic regional pain is found in $20 \%$ to $25 \%$ of the population. Both chronic widespread and regional pain occur about 1.5 times as commonly in women than men. Between $25 \%$ and $50 \%$ of individuals who have CWP will also have 11 or greater tender points, and thus meet criteria for fibromyalgia (Jacobsen and Bredkjaer 1992; Cöster et al 2007). The prevalence of fibromyalgia is just as high in rural or nonindustrialized societies as it is in countries such as the US (Raspe 1992; White and Thompson 2003; Peleg et al 2007).

In summary, although many clinicians uniquely associate fibromyalgia with women who display high levels of distress, much of this is an artifact of: 1) the ACR criteria that require 11 tender points, and 2) the fact that most studies of fibromyalgia have originated from clinical samples from tertiary care centers, where healthcare seeking behaviors lead to the fact that psychological and psychiatric co-morbidities are much higher (Aaron et al 1996). When all these biases are eliminated by examining CWP in population-based studies, a clearer picture of fibromyalgia is obtained, and chronic widespread pain becomes much like chronic musculoskeletal pain in any other region of the body.

\section{Etiology \\ Genetic factors}

Research has indicated a strong familial component to the development of fibromyalgia. First degree relatives of individuals with fibromyalgia display an eight-fold greater risk of developing fibromyalgia than those in the general population (Arnold et al 2004a). These studies also show that family members of individuals with fibromyalgia are much more tender than the family members of controls, regardless of whether they have pain or not. Family members of fibromyalgia patients are also much more likely to have IBS, TMD, headaches, and a host of other regional pain syndromes (Hudson et al 1985; Buskila et al 1996; Kato et al 2006b). This familial and personal co-aggregation of conditions which includes fibromyalgia was originally collectively termed affective spectrum disorder, (Hudson et al 1993) and more recently central sensitivity syndromes and chronic multisymptom illnesses (Fukuda et al 1998; Yunus 2008). In population-based studies, the key symptoms that often co-aggregate besides pain are fatigue, memory difficulties, and mood disturbances (Fukuda et al 1997, 1998). Twin studies suggest that approximately half of the risk of developing CWP is due to genetic factors, and half environmental (Kato et al 2006a).

Recent studies have begun to identify specific genetic polymorphisms that are associated with a higher risk of developing fibromyalgia. To date, the serotonin 5-HT2A receptor polymorphism $\mathrm{T} / \mathrm{T}$ phenotype, serotonin transporter, dopamine 4 receptor, and COMT (catecholamine $o$-methyl transferase) polymorphisms have all been noted to be seen in higher frequency in fibromyalgia (Bondy et al 1999; Offenbaecher et al 1999; Buskila et al 2004; Buskila 2007). It is of note that all of the polymorphisms identified to date involve the metabolism or transport of monoamines, compounds that play a critical role in activity of the human stress response. However these trials have used a targeted approach where a specific gene polymorphism has been examined. It is likely that there are scores of other genetic polymorphisms, involving other neuromodulators as well as monoamines, which in part determine an individuals" "set point" for pain and sensory processing.

\section{Environmental factors}

As with most illnesses that may have a genetic underpinning, environmental factors may play a prominent role in triggering the development of fibromyalgia and related conditions. Environmental "stressors" temporally associated with the development of either fibromyalgia or chronic fatigue syndrome includes physical trauma, certain infections such as Hepatitis C, Epstein Barr virus, parvovirus, Lyme disease, and emotional stress. The disorder is also associated with other regional pain conditions or autoimmune disorders (Buskila et al 1997; Clauw and Chrousos 1997; McLean and Clauw 2004). Of note, each of these stressors only 
leads to CWP or fibromyalgia in approximately 5\%-10\% of individuals who are exposed; the overwhelming majority of individuals who experience these same infections or other stressful events regain their baseline state of health.

An excellent recent example of how illnesses such as fibromyalgia might be triggered occurred in the setting of the deployment of troops to liberate Kuwait during the Gulf War in 1990 and 1991. The term "Gulf War illnesses" is now commonly used to refer to a constellation of symptoms developed by some $10 \%-15 \%$ of the 700,000 US troops deployed to the Persian Gulf in the early 1990s. The symptoms, which include headaches, muscle and joint pain, fatigue, memory disorders and gastrointestinal distress (Fukuda et al1998), were seen in troops deployed from the United Kingdom (UK) and other countries as well (Unwin et al 1999). The panels of experts who examined potential causes for these symptoms and syndromes found that the sickness could not be traced to any single environmental trigger, and noted the similarities between these individuals, and those diagnosed with fibromyalgia and chronic fatigue. Furthermore, similar syndromes involving multiple somatic symptoms have been noted in veterans of every war the US or UK has been involved in during the past century (Hyams et al 1996). This suggests that war may be an environment where individuals are simultaneously exposed to a multitude of "stressors. triggering the development of this type of illness in susceptible individuals (Clauw 2003).

The above-noted relationship between individuals with other chronic rheumatic or autoimmune disorders deserves special attention, because of the relevance to practicing clinicians. As many as $25 \%$ of patients correctly diagnosed with generalized inflammatory disorders such as systemic lupus erythmatosus (SLE), rheumatoid arthritis (RA), and ankylosing spondylitis will also fulfill ACR criteria for fibromyalgia (Clauw and Katz 1995). However, in clinical practice this co-expression may go unrecognized, especially when the fibromyalgia develops after the autoimmune disorder or regional pain syndrome. In this setting when co-morbid fibromyalgia goes unrecognized, patients are often unnecessarily treated more aggressively with toxic immunosuppressive drugs.

\section{Pathogenesis \\ Role of stressors}

Once FIBROMYALGIA develops, the mechanisms responsible for ongoing symptom expression are likely complex and multifactorial. Because of the fact that disparate "stressors" can trigger the development of these conditions, the human stress response has been closely examined for a causative role. These systems are mediated primarily by the activity of the corticotropin-releasing hormone $(\mathrm{CRH})$ nervous system located in the hypothalamus and locus-ceruleus-norepinephrine/autonomic (sympathetic/ LC-NE) nervous system in the brain stem. Recent research suggests that although this system in humans has been highly adaptive throughout history, the stress response may be inappropriately triggered by a wide assortment of everyday occurrences that do not pose a real threat to survival, thus initiating the cascade of physiologic responses more frequently than can be tolerated (Sapolsky 1996).

The type of stress and the environment in which it occurs also have an impact on how the stress response is expressed. An animal study has shown that that the strongest physiological responses are triggered by events that are accompanied by a lack of control or support, and thus viewed as perceived as inescapable or unavoidable (Chrousos and Gold 1992). In humans, daily "hassles" and personally relevant stressors seem to be more capable of causing symptoms, which may lead to fibromyalgia, than major catastrophic events that do not personally impact on the individual (Pillow et al 1996).

Two studies performed in the US just before and after the terrorist attacks of 9/11 point out that not all psychological stress is capable of triggering or exacerbating fibromyalgia or somatic symptoms. In one study performed by Raphael and colleagues (2002), no difference in pain complaints or other somatic symptoms was seen in residents of New York and New Jersey who had been surveyed prior to $9 / 11$, and then just following the terrorist attacks on the World Trade Center. In another study performed in the Washington DC region during the same time period, patients with fibromyalgia had no worsening of pain or other somatic symptoms following the attacks, compared to just before the attack (Williams et al 2003).

Recent reviews regarding the role that "stressors" or catastrophic events may have in triggering the development of fibromyalgia or related conditions have identified a number of factors that may be much more important than the intensity of the "stressor" in predicting adverse health outcomes. Female gender, worry or expectation of chronicity, and inactivity or time off work following the stressor make it more likely to trigger the development of pain or other somatic symptoms (McLean and Clauw 2004). Naturally occurring catastrophic events such as earthquakes, floods, or fires are much less likely to lead to chronic somatic symptoms than similarly stressful events that are "man made" such as chemical spills, or war (Clauw et al 2003). Being exposed to a 
multitude of stressors simultaneously, or over a period of time, may also be a significant risk for later somatic symptoms and or psychological sequelae. Intensely stressful events can lead to permanent changes in the activity of both mouse and human stress response systems (Sapolsky 1996; Heim et al 2001).

To complete this vicious circle, these changes in baseline function of the stress response that may occur following a stressor earlier in life have been shown to predict which symptom-free individuals without chronic pain or other somatic symptoms are more likely to develop these somatic symptoms. This has been noted both in populationbased studies and in experiments where healthy young adults are deprived of regular sleep or exercise (Glass et al 2004; McBeth et al 2007).

This theoretical link between stress, changes in stress axis activity, and subsequent susceptibility to develop somatic symptoms or syndromes is also supported by studies showing that patients with fibromyalgia and related conditions may be more likely than nonaffected individuals to have experienced physical or sexual abuse in childhood (Boisset-Pioro et al 1995; Drossman 1995; Aaron et al 1997; Alexander et al 1998). Twin studies have recently supported a link between post-traumatic stress disorder (PTSD) and trauma, and CWP (Arguelles et al 2006). Just as a lack of or cessation of exercise following trauma seems to be associated with a higher likelihood of developing pain or other somatic symptoms, a recent study of Israeli war veterans with PTSD showed that those who exercised regularly were much less likely to develop CWP or fibromyalgia (Arnson et al 2007).

\section{Augmented pain and sensory processing as a hallmark of fibromyalgia and related syndromes}

Once fibromyalgia is established, by far the most consistently detected objective abnormalities involve pain and sensory processing systems. Since fibromyalgia is defined in part by tenderness, considerable work has been performed exploring the potential reason for this phenomenon. The results of two decades of psychophysical pressure pain testing in fibromyalgia have been very instructive.

One of the earliest findings in this regard was that the tenderness in fibromyalgia is not confined to tender points, but instead extends throughout the entire body (Granges and Littlejohn 1993; Wolfe et al 1995). Theoretically, such diffuse tenderness could be either primarily due to psychological (eg, hypervigilance, where individuals are too attentive to their surroundings), or neurobiological factors.
Early studies typically used dolorimetry to assess pressure pain threshold, and concluded that tenderness was in large part related to psychological factors, because these measures of pain threshold were correlated with levels of distress (Wolfe et al 1995; Wolfe 1997; Gracely et al 2003). Also, nuances such as the rate of increase of stimulus pressure, controlled by the operator vs. by the patient, and patient distress have been shown to influence pain threshold when it is measured in this manner (Jensen et al 1986; Petzke et al 2003b).

To minimize the biases associated with "ascending" (ie, the individual knows that the pressure will be predictably increased) measures of pressure pain threshold, Petzke and colleagues (1999, 2001, 2003a) performed a series of studies using more sophisticated paradigms using random delivery of pressures. These studies showed that: 1) the random measures of pressure pain threshold were not influenced by levels of distress of the individual, whereas tender point count and dolorimetry exams were, 2) fibromyalgia patients were much more sensitive to pressure even when these more sophisticated paradigms were used, 3) fibromyalgia patients were not any more "expectant" or "hypervigilant" than controls, and 4) pressure pain thresholds at any four points in the body are highly correlated with the average tenderness at all 18 tender points and four "control points" (the thumbnail and forehead).

Because of this close link between tenderness and fibromyalgia, this phenomenon has also been well studied as a potential relevant outcome in clinical trials of new fibromyalgia therapies. In a number of longitudinal randomized placebo controlled trials fibromyalgia, improvements in clinical pain have corresponded with a significant change in tender point counts or tender point index (Farber et al 2000). In contrast, other studies did not show a correspondence between improvements in clinical pain and tender point counts (Scudds et al 1989; Jacobsen et al 1991; Goldenberg et al 1996; Arnold et al 2000, 2002; Gowans et al 2001). These discrepancies between previous studies could either be because these therapies did not improve tenderness, or because tender points are not a good measure of tenderness. Two recent studies suggest the latter, since when individuals with fibromyalgia were simultaneously assessed using tender point counts, dolorimetry, and random pressure paradigms, the random pressure paradigms showed the most responsiveness to change (Harris et al 2006; Geisser et al 2007).

\section{Specific mechanisms that may lead to a low pain threshold in fibromyalgia}

There are two different specific pathogenic mechanisms in fibromyalgia that have been identified using experimental 
pain testing: 1) an absence or decrease of descending analgesic activity, and 2) increased wind-up or temporal summation.

\section{Attenuated diffuse noxious inhibitory controls (DNIC) in fibromyalgia}

In healthy humans and laboratory animals, application of an intense painful stimulus for two to five inutes produces generalized whole-body analgesia. This analgesic effect, termed "diffuse noxious inhibitory controls" (DNIC), has been consistently observed to be attenuated or absent in groups of fibromyalgia patients, compared to healthy controls (Kosek and Hansson 1997; Lautenbacher and Rollman 1997; Leffler et al 2002; Julien et al 2005). Wilder-Smith and Robert-Yap (2007) have performed studies suggesting that in IBS there is a similar decrease in descending analgesic activity. A point of emphasis is that this finding of attenuated DNIC is not found in all fibromyalgia or IBS patients, but is considerably more common in patients than controls.

The DNIC response in humans is believed to be partly mediated by descending opioidergic pathways and in part by descending serotonergic-noradrenergic pathways. In fibromyalgia, the accumulating data suggests that oioidergic activity is abnormal in fibromyalgia, in that levels of cerebrospinal fluid (CSF) enkephalins are roughly twice as high in fibromyalgia and idiopathic low back pain patients as in healthy controls (Baraniuk et al 2004). Moreover, PET data show that baseline mu-opioid receptor binding is decreased in multiple pain processing regions in the brains of fibromyalgia patients, consistent with either increased release of endogenous mu-opioid ligands in fibromyalgia or reduced numbers of receptors (Harris et al 2007).

The biochemical and imaging findings suggesting altered activity of endogenous opioidergic systems in fibromyalgia are consistent with the anecdotal experience that opioids are generally ineffective analgesics in patients with fibromyalgia and related conditions. In contrast, studies have shown the opposite for serotonergic and noradrenergic activity in fibromyalgia. Studies have shown that the principal metabolite of norepinephrine, 3-methoxy4-hydroxyphenethylene (MPHG), is lower in the CSF of fibromyalgia patients (Russell et al 1992). Similarly, there are data suggesting low serotonin in this syndrome. Patients with fibromyalgia were shown to have reduced serum levels of serotonin and its precursor, L-tryptophan, as well as reduced levels of the principal serotonin metabolite, 5-HIAA, in their CSF (Russell et al 1992; Yunus et al 1992). Further evidence for this mechanism comes from treatment studies, where nearly any type of compound that simultaneously raises both serotonin and norepinephrine has been shown to be efficacious in treating fibromyalgia and related conditions (Arnold et al 2000, 2004b; Bennett et al 2003; Gendreau et al 2005).

Another important neurotransmitter in pain processing, and one that likely is playing some role in fibromyalgia, is glutamate. Glutamate is a major excitatory neurotransmitter within the central nervous system, and cerebrospinal fluid levels of glutamate are twice as high in fibromyalgia patients than controls (Sarchielli et al 2007). Not only are these levels elevated, but a recent study using proton spectroscopy demonstrated that the glutamate levels in the insula in fibromyalgia change in response to changes in both clinical and experimental pain when patients are treated with acupuncture or sham acupuncture (Harris et al 2008).

Thus, a number of lines of evidence point to the fact that fibromyalgia is a state of heightened pain or sensory processing, and that this might occur because of high levels of neurotransmitters that increase pain transmission, and/or low levels of neurotransmitters that decrease pain transmission.

\section{Abnormalities on functional neuroimaging}

Functional neural imaging enables investigators to visualize how the brain processes the sensory experience of pain. The primary modes of functional imaging that have been used in fibromyalgia include functional magnetic resonance imaging (fMRI), single photon emission computed tomography (SPECT), and positron emission tomography (PET), and proton spectroscopy (H-MRS).

\section{Single photon emission computed tomography}

Single photon emission computed tomography (SPECT) was the first functional neuroimaging technique to be used in fibromyalgia. SPECT imaging involves the introduction of radioactive compounds into the participant's blood stream, which then decay over time giving a window for neural activity assessment. The first trial using SPECT imaging in fibromyalgia patients was conducted by Mountz and colleagues (1995). Their data from 10 fibromyalgia patients and seven age- and education-matched healthy controls indicated that both the caudate and the thalamus of fibromyalgia patients had decreased blood flow. The findings by Mountz and colleagues (1995) were largely replicated in a second SPECT study by Kwiatek and colleagues (2000). In a third SPECT trial, Guedj and colleagues (2007a, 2007b) reported a study using a more sensitive radioligand (99mTc-ECD) in fibromyalgia patients and pain free 
controls. Guedj and colleagues found hyperperfusion in fibromyalgia patients within the somatosensory cortex and hypoperfusion in the anterior and posterior cingulate, the amygdala, medial frontal and parahippocampal gyrus, and the cerebellum. Finally, if these regional cerebral blood flow (rCBF) differences are relevant for fibromyalgia pathology, one could hypothesize that changes in $\mathrm{rCBF}$ should track with changes in pain symptoms over time. One longitudinal treatment trial used SPECT imaging to assess changes in rCBF following administration of amitriptyline within 14 fibromyalgia patients (Adiguzel et al 2004). After three months of treatment with amitriptyline, increases in $\mathrm{rCBF}$ in the bilateral thalamus and the basal ganglia were observed. Since the same two regions had been implicated previously, these data suggest that amitriptyline may normalize the altered blood flow which is associated with reduced pain symptoms.

\section{Functional MRI (fMRI)}

fMRI is a noninvasive brain imaging technique that relies on changes in the relative concentration of oxygenated to deoxygenated hemoglobin within the brain. In response to neural activity, oxygenated blood flow is increased within the local brain area. This causes a decrease in the concentration of deoxygenated hemoglobin. Since deoxygenated hemoglobin is paramagnetic, this in turn causes a change in the magnetic property of the tissue. Unlike SPECT and PET which can measure baseline levels of blood flow, the fMRI blood oxygenation level dependent (BOLD) effect originates from a difference between experimental conditions and does not assess baseline blood flow. Typically in fibromyalgia trials involving fMRI, evoked pain sensations are compared to "off" conditions that have either no pain or involve an innocuous sensation.

The first study to use fMRI in fibromyalgia patients was performed by Gracely and colleagues (2002) In this study 16 fibromyalgia patients and 16 matched controls were exposed to painful pressures during the fMRI experiment. The authors found increased neural activations (ie, increases in the BOLD signal) in patients compared to pain-free controls, when stimuli of equal pressure magnitude were administered. Regions of increased activity included the primary and secondary somatosensory cortex, the insula, and the anterior cingulate, all regions commonly observed in fMRI studies of healthy normal subjects during painful stimuli. Interestingly, when the pain-free controls were subjected to pressures that evoked equivalent pain ratings in the fibromyalgia patients, similar activation patterns were observed. These findings were entirely consistent with the "left-shift" in stimulus-response function noted with experimental pain testing, and suggest that fibromyalgia patients experience an increased gain or "volume setting" in brain sensory processing systems. In a similar experiment, Cook and colleagues used painful heat stimuli during fMRI (Cook et al 2004b). Similar to the Gracely and colleagues' findings, the authors observed significant increases in the pain ratings of patients and augmented pain processing within the contralateral insula. fMRI has also proved useful in determining how co-morbid psychological factors influence pain processing in fibromyalgia. For example, a recent study by Giesecke and colleagues (2005) explored the relationship between depression and enhanced evoked pain sensations in 30 patients with fibromyalgia. The authors found that the anterior insula and amygdala activations were correlated with depressive symptoms, consistent with these regions being involved with affective or motivational aspects of pain processing. However, the degree of neuronal activation in areas of the brain thought to be associated with the "sensory" processing of pain (ie, where the pain is localized and its intensity) were not associated with levels of depressive symptoms, or the presence or absence of major depression. These data are consistent with a plethora of evidence in the pain field that there are different regions of the brain responsible for pain processing devoted to sensory intensity versus affective aspects of pain sensation, and suggest that the former and latter are largely independent of each other. In contrast, this same group showed that the presence of catastrophizing, a patient's negative or pessimistic appraisal of their pain, influences both the sensory and affective dimensions of pain on fMRI in fibromyalgia (Gracely et al 2004).

PET has been used in a few studies in fibromyalgia. In the first such study, Yunus and colleagues (1997, 2004) did not identify any differences in regional cerebral blood flow between fibromyalgia patients and controls. However, Wood and colleagues used PET to show that attenuated dopaminergic activity may be playing a role in pain transmission in fibromyalgia, and Harris and colleagues showed evidence of decreased $m u$ opiod receptor availability (possibly due to increased release of endogenous $m u$ opioids) in fibromyalgia (Harris et al 2007).

\section{Treatment}

Progress in the understanding of fibromyalgia has led to more therapeutic options for patients with this condition. Investigators are examining the utility of newer medications 
as well as nonpharmacological interventions in controlled trials. Clinical based evidence advocates a multi-faceted program emphasizing education, certain medications, exercise, and cognitive therapy (Goldenberg et al 2004).

\section{Pharmacologic therapy}

The majority of fibromyalgia clinical trials have involved antidepressants of one class or another. Trials studying the oldest class of agents, tricyclic antidepressants (TCAs), are most abundant, though several recent studies have focused on selective serotonin reuptake inhibitors and "atypical antidepressants", a class that includes dual reuptake inhibitors and monoamine oxidase inhibitors (MAOIs).

\section{Tricyclic anitdepressants}

The most frequently studied pharmacologic therapy for fibromyalgia is low doses of tricyclic compounds. Most TCAs increase the concentrations of serotonin and/or norepinephrine (noradrenaline) by directly blocking their respective reuptake. The effectiveness of TCAs, particularly amitriptyline and cyclobenzaprine, in treating the symptoms of pain, poor sleep, and fatigue associated with fibromyalgia is supported by several randomized, controlled trials (Arnold et al 2000). Tolerability is a problem but can be improved by beginning at very low doses (eg, $10 \mathrm{mg}$ of amitriptyline), giving the dose a few hours before bedtime, and very slowly escalating the dose.

\section{Selective serotonin reuptake inhibitors}

Because of a better side effect profile, newer antidepressants, ie, selective serotonin reuptake inhibitors (SSRIs), are frequently used in fibromyalgia. The SSRIs fluoxetine, citalopram, and paroxetine have each been evaluated in randomized, placebo controlled trials (Norregaard et al 1995; Anderberg et al 2000; Capaci and Hepguler 2002; Goldenberg et al 2004). In general, the results of studies of SSRIs in fibromyalgia have paralleled the experience in other pain conditions. The newer "highly selective" serotonin reuptake inhibitors such as citalopram, seem to be less efficacious than the older SSRIs, which have some noradrenergic activity at higher doses (Fishbain 2000).

Since TCAs, and high doses of certain "SSRIs" such as fluoxetine and sertraline, that have the most balanced re-uptake inhibition are the most effective analgesics, many have concluded that dual receptor inhibitors such as serotonin-NE and NE-serotonin reuptake inhibitors (SNRIs and NSRIs) may be of more benefit than pure serotonergic drugs (Fishbain 2000). These drugs are pharmacologically similar to some TCAs in their ability to inhibit the reuptake of both serotonin and NE, but differ from TCAs in being generally devoid of significant activity at other receptor systems. This selectivity results in diminished side effects and enhanced tolerability. The first available SNRI, venlafaxine, has data to support its use in the management of neuropathic pain, and retrospective trial data demonstrate that this compound is also effective in the prophylaxis of migraine and tension headaches (Adelman et al 2000). Two studies in fibromyalgia have had conflicting results, with the one using a higher dose showing efficacy (Goldenberg et al 2004).

\section{Milnacipran and duloxetine}

Two new SNRIs, milnacipran and duloxetine, have undergone recent multicenter trials and were shown to be effective in a number of outcome variables (Arnold et al 2004b; Vitton et al 2004). These newer drugs have promise for reducing pain symptoms in fibromyalgia. In the Vitton and colleagues (2004) study evaluating milnacipran, statistically significant differences were noted in overall improvement, physical functioning, level of fatigue and degree of reported physical impairment. In the Arnold and colleagues (2004b) trial of duloxetine compared to placebo, participants treated with duloxetine had decreased self-reported pain and stiffness, and a reduced number of tender points. In both trials, benefits were shown to be independent of the drug effect on mood, thus suggesting that the analgesic and other positive effects of milnacipran and duloxetine in fibromyalgia are not simply due to their antidepressant effects. Also both studies found these drugs to be generally well tolerated, with side effects commonly seen with this class of drugs such as nausea and palpitations, as well as mild changes in pulse and blood pressure in some patients.

Within the Vitton and colleagues (2004) study, the analgesic response seen with milnacipran was rapid and sustained. However, it is clear that this drug is not simply acting as an analgesic, because there were parallel changes seen in other domains such as depression, fatigue, and cognition over the course of the study. These improvements in fatigue and cognition may be due to the fact that milnacipran has proportionally greater noradrenergic than serotinergic re-uptake effects, when compared with other dual re-uptake inhibitors.

It is unlikely that the beneficial effects of milnacipran are due to its antidepressant properties (Briley et al 1996). This trial excluded patients with major depression, and in spite of this improvement efficacy was seen across a number of domains. Other studies of dual re-uptake inhibitors such as tricyclics 
and duloxetine have similarly shown that the analgesic efficacy of these compounds across a variety of pain conditions is similar in depressed and nondepressed individuals (Arnold 2007; Fishbain 2000). These findings are also consistent with current theories of pain processing which suggest that the sensory processing of pain (ie, where it is located and how much it hurts) occurs in different brain regions than the affective and cognitive processing of pain (ie, the emotional valence and cognitive appraisal of pain) (Derbyshire 2000). In fact, functional neuroimaging studies in fibromyalgia have identified augmented central pain processing in the regions of the brain associated with the sensory and affective processing of pain, and have demonstrated that the magnitude of neuronal hyperactivity seen with painful stimuli in the sensory regions (eg, the primary and secondary somatosensory cortices) is independent of whether the fibromyalgia patient is depressed or not (Gracely et al 2002; Cook et al 2004a; Giesecke, et al 2005).

The fact that a drug that inhibits the reuptake of serontonin and norepinephrine is efficacious in fibromyalgia is not surprising. There are abundant data suggesting that at least a subset of fibromyalgia patients has a deficiency in serotinergic and noradrenergic activity that is responsible for their pain, tenderness, and other symptoms. Studies have shown that the principal metabolite of norepinephrine, 3-methoxy-4-hydroxyphenethylene (MPHG), is lower in the CSF of fibromyalgia patients (Russell et al 1992). Similarly, there are data suggesting low serotonin in this syndrome. Patients with fibromyalgia have been shown to have reduced serum levels of serotonin and its precursor, L-tryptophan, as well as reduced levels of the principal serotonin metabolite, 5-HIAA, in their CSF (Russell et al 1992; Yunus et al 1992).

The prevailing theory is that this decreased serotinergic and noradrenergic activity leads to a decrease in activity via descending analgesic pathways, which in turn leads to the pain as well as diffuse hyperalgesia and allodynia seen in fibromyalgia and a number of related conditions. As mentioned above, in healthy humans and laboratory animals, application of an intense painful stimulus for two to five minutes produces generalized whole-body analgesia, termed "diffuse noxious inhibitory controls" (DNIC). This is believed to be partly mediated by descending opioidergic pathways and in part by descending serotonergicnoradrenergic pathways. DNIC has been consistently observed to be attenuated or absent in groups of fibromyalgia patients, compared to healthy controls (Kosek and Hansson 1997; Lautenbacher and Rollman 1997; Leffler et al 2002;
Julien et al 2005). In fibromyalgia, the accumulating data suggests that opioidergic activity is abnormal, in that levels of cerebrospinal fluid (CSF) enkephalins are roughly twice as high in fibromyalgia and idiopathic low back pain patients as in healthy controls (Baraniuk et al 2004). Moreover, positron emission tomography data show that baseline mu-opioid receptor binding is decreased in multiple pain processing regions in the brains of fibromyalgia patients, consistent with the hypothesis that there is increased release of endogenous mu-opioid ligands or reduced receptor number in fibromyalgia leading to high baseline occupancy of the receptors (Harris et al 2007). Thus, although it has not been definitively proven that compounds that raise both serotonin and norepinephrine act as analgesics by restoring descending analgesic activity in fibromyalgia and a number of other chronic pain states, there is compelling evidence that this is likely to be the case.

In addition to leading to improvements in a number of symptoms, administration of milnacipran was also associated with improvements in functional status (Vitton et al 2004). This is important, since the functional status of fibromyalgia patients is very impaired, compared to healthy controls (Kop et al 2005). It is likely that much greater improvements in functional status would be noted if patients were given non-pharmacological therapies such as exercise and cognitive behavioral therapy as well as milnacipran or other drugs, as these nondrug therapies typically have more robust effects on function (O’Malley et al 2000).

Although milnacipran was generally well tolerated, approximately twice as many individuals in the active treatment group withdrew from the study because of adverse effects as those in the placebo-treated group (Vitton et al 2004). The most common side effects seen with the drug were nausea, constipation, palpitations, and flushing. These side effects are typically noted with this class of drugs, and these symptoms often resolve within days to weeks. These side effects also can be reduced if this class of drug is taken with food and the dose is escalated slowly. There were also notable effects on both pulse and blood pressure seen in a small percentage of individuals receiving milnacipran, presumably due to the noradrenergic effects, so individuals should be monitored for these side effects.

In conclusion, milnacipran dosed BID at 100 or $200 \mathrm{mg}$ per day led to global improvements in fibromyalgia, characterized by relief of pain, fatigue, and other symptoms (Vitton et al 2004). Further studies will help clarify the precise role of this and other compounds in treating fibromyalgia in clinical practice. Like many drugs used for many diseases, 
this compound clearly does not work in all individuals with fibromyalgia, which should not be surprising given that emerging evidence suggests that this is a polygenic illness with many root causes (Arnold et al 2004a; Buskila et al 2007). It may often be necessary to use this compound in combination with other drugs with different mechanisms of action, and with nondrug therapies such as exercise and cognitive behavioral therapy, to achieve a more complete response in most fibromyalgia patients.

\section{Disclosure}

The authors report no conflicts of interest in this work.

\section{References}

Aaron LA, Bradley LA, Alarcon GS, et al. 1996. Psychiatric diagnoses in patients with fibromyalgia are related to health care-seeking behavior rather than to illness. Arthritis Rheum, 39:436-45.

Aaron LA, Bradley LA, Alarcon GS, et al. 1997. Perceived physical and emotional trauma as precipitating events in fibromyalgia. Associations with health care seeking and disability status but not pain severity. Arthritis Rheum, 40:453-60.

Adelman LC, Adelman JU, Von Seggern R, et al. 2000. Venlafaxine extended release (XR) for the prophylaxis of migraine and tension-type headache: A retrospective study in a clinical setting. Headache, 40:572-80.

Adiguzel O, Kaptanoglu E, Turgut B, et al. 2004. The possible effect of clinical recovery on regional cerebral blood flow deficits in fibromyalgia: a prospective study with semiquantitative SPECT. South Med J, 97:651-55.

Alexander RW, Bradley LA, Alarcon GS, et al. 1998. Sexual and physical abuse in women with fibromyalgia: association with outpatient health care utilization and pain medication usage. Arthritis Care Res, 11:102-15.

Anderberg UM, Marteinsdottir I, Von Knorring L. 2000. Citalopram in patients with fibromyalgia - a randomized, double-blind, placebocontrolled study. Eur J Pain, 4:27-35.

Arguelles LM, Afari N, Buchwald DS, et al. 2006. A twin study of posttraumatic stress disorder symptoms and chronic widespread pain. Pain, 124:150-7.

Arnold LM. 2007. Duloxetine and other antidepressants in the treatment of patients with fibromyalgia. Pain Med, 8:S63-S74.

Arnold LM, Hess EV, Hudson JI, et al. 2002. A randomized, placebocontrolled, double-blind, flexible-dose study of fluoxetine in the treatment of women with fibromyalgia. Am J Med, 112:191-7.

Arnold LM, Hudson JI, Hess EV, et al. 2004a. Family study of fibromyalgia. Arthritis Rheum, 50:944-52.

Arnold LM, Keck PEJ, Welge JA. 2000. Antidepressant treatment of fibromyalgia. A meta-analysis and review. Psychosomatics, 41:104-13.

Arnold LM, Lu Y, Crofford LJ, et al. 2004b. A double-blind, multicenter trial comparing duloxetine with placebo in the treatment of fibromyalgia patients with or without major depressive disorder. Arthritis Rheum, 50:2974-84

Arnson Y, Amital D, Fostick L, et al. 2007. Physical activity protects male patients with post-traumatic stress disorder from developing severe fibromyalgia. Clin Exp Rheumatol, 25:529-33.

Baraniuk JN, Whalen G, Cunningham J, et al. 2004. Cerebrospinal fluid levels of opioid peptides in fibromyalgia and chronic low back pain. BMC Musculoskelet Disord, 5:48.

Barsky AJ, Borus JF. 1999. Functional somatic syndromes. Ann Intern Med, 130:910-21.

Bennett RM, Kamin M, Karim R, et al. 2003. Tramadol and acetaminophen combination tablets in the treatment of fibromyalgia pain: a doubleblind, randomized, placebo-controlled study. Am J Med, 114:537-45.
Boisset-Pioro MH, Esdaile JM, Fitzcharles MA. 1995. Sexual and physical abuse in women with fibromyalgia syndrome. Arthritis Rheum, 38:235-41.

Bondy B, Spaeth M, Offenbaecher M, et al. 1999. The T102C polymorphism of the 5-HT2A-receptor gene in fibromyalgia. Neurobiol Dis, 6:433-9.

Briley M, Prost JF, Moret C. 1996. Preclinical pharmacology of milnacipran. Int Clin Psychopharmacol, 11:9-14.

Buskila D. 2007. Genetics of chronic pain states. Best Pract Res Clin Rheumatol, 21:535-47.

Buskila D, Cohen H, Neumann L, et al. 2004. An association between fibromyalgia and the dopamine D4 receptor exon III repeat polymorphism and relationship to novelty seeking personality traits. Mol Psychiatry, 9:730-1.

Buskila D, Neumann L, Hazanov I, et al. 1996. Familial aggregation in the fibromyalgia syndrome. Semin Arthritis Rheum, 26:605-11.

Buskila D, Neumann L, Vaisberg G, et al. 1997. Increased rates of fibromyalgia following cervical spine injury. A controlled study of 161 cases of traumatic injury. Arthritis Rheum, 40:446-52.

Buskila D, Sarzi-Puttini P, Ablin JN. 2007. The genetics of fibromyalgia syndrome. Pharmacogenomics, 8:67-74.

Capaci K, Hepguler S. 2002. Comparison of the effects of amitriptyline and paroxetine in the treatment of fibromyalgia syndrome. The Pain Clinic, 14:223-8.

Chrousos GP, Gold PW. 1992. The concepts of stress and stress system disorders. Overview of physical and behavioral homeostasis. JAMA, 267:1244-52.

Clauw DJ. 2003. The health consequences of the first Gulf war. Br Med J, $327: 1357-8$

Clauw DJ. 2007. Fibromyalgia: update on mechanisms and management. J Clin Rheumatol, 13:102-9.

Clauw DJ, Chrousos GP. 1997. Chronic pain and fatigue syndromes: Overlapping clinical and neuroendocrine features and potential pathogenic mechanisms. Neuroimmunomodulation, 4:134-53.

Clauw DJ, Engel CC Jr, Aronowitz R, et al. 2003. Unexplained symptoms after terrorism and war: an expert consensus statement. JOccup Environ Med, 45:1040-8.

Clauw DJ, Katz P. 1995. The overlap between fibromyalgia and inflammatory rheumatic diseases: When and why does it occur? J Clin Rheum, 1:335-41.

Cook DB, Lange G, Ciccone DS, et al. 2004a. Functional imaging of pain in patients with primary fibromyalgia. $J$ Rheumatol, 31:364-78.

Cöster L, Kendall S, Gerdle B, et al. 2007. Chronic widespread musculoskeletal pain - A comparison of those who meet criteria for fibromyalgia and those who do not. Eur J Pain, 12:600-10.

Croft P, Rigby AS, Boswell R, et al. 1993. The prevalence of chronic widespread pain in the general population. $J$ Rheumatol, 20:710-3.

Derbyshire SW. 2000. Exploring the pain "neuromatrix". Curr Rev Pain, 4:467-77.

Drossman DA. 1995. Sexual and physical abuse and gastrointestinal illness. Scand J Gastroenterol Suppl, 208; 90-96.

Drossman DA, Li ZM, Andruzzi E, et al. 1993. US householder survey of functional gastrointestinal disorders. Prevalence, sociodemography, and health impact. Dig Dis Sci, 38:1569-80.

Farber L, Stratz T, Bruckle W, et al. 2000. Efficacy and tolerability of tropisetron in primary fibromyalgia - A highly selective and competitive 5-HT3 receptor antagonist. German Fibromyalgia Study Group. Scand J Rheum Suppl, 113:49-54.

Fishbain D. 2000. Evidence-based data on pain relief with antidepressants. Ann Med, 32:305-16.

Fukuda K, Dobbins JG, Wilson LJ, et al. 1997. An epidemiologic study of fatigue with relevance for the chronic fatigue syndrome. $J$ Psychiatr Res, 31:19-29.

Fukuda K, Nisenbaum R, Stewart G, et al. 1998. Chronic multisymptom illness affecting Air Force veterans of the Gulf War. JAMA, 280:981-8.

Geisser ME, Gracely RH, Giesecke T, et al. 2007. The association between experimental and clinical pain measures among persons with fibromyalgia and chronic fatigue syndrome. Eur J Pain, 11:202-7. 
Gendreau RM, Thorn MD, Gendreau JF, et al. 2005. The efficacy of milnacipran in fibromyalgia. $J$ Rheumatol, 32:1975-85.

Giesecke J, Reed BD, Haefner HK, et al. 2004a. Quantitative sensory testing in vulvodynia patients and increased peripheral pressure pain sensitivity. Obstet Gynecol, 104:126-33.

Giesecke T, Gracely RH, Williams DA, et al. 2005. The relationship between depression, clinical pain, and experimental pain in a chronic pain cohort. Arthritis Rheum, 52:1577-84.

Giesecke T, Gracely RH, Grant MA, et al. 2004b. Evidence of augmented central pain processing in idiopathic chronic low back pain. Arthritis Rheum, 50:613-23.

Glass JM, Lyden A, Petzke F, et al. 2004. The effect of brief exercise cessation on pain, fatigue, and mood symptom development in healthy, fit individuals. $J$ Psychosom Res, 57:391-8.

Goldenberg D, Mayskiy M, Mossey C, et al. 1996. A randomized, double-blind crossover trial of fluoxetine and amitriptyline in the treatment of fibromyalgia. Arthritis Rheum, 39:1852-9.

Goldenberg DL, Burckhardt C, Crofford L. 2004. Management of fibromyalgia syndrome. JAMA, 292:2388-95.

Gowans SE, de Hueck A, Voss S, et al. 2001. Effect of a randomized, controlled trial of exercise on mood and physical function in individuals with fibromyalgia. Arthritis Rheum, 45:519-29.

Gracely RH, Geisser ME, Giesecke T, et al. 2004. Pain catastrophizing and neural responses to pain among persons with fibromyalgia. Brain, 127(Pt 4):835-43.

Gracely RH, Grant MA, Giesecke T. 2003. Evoked pain measures in fibromyalgia. Best Pract Res Clin Rheumatol, 17:593-609.

Gracely RH, Petzke F, Wolf JM, et al. 2002. Functional magnetic resonance imaging evidence of augmented pain processing in fibromyalgia. Arthritis Rheum, 46:1333-43.

Granges G, Littlejohn G. 1993. Pressure pain threshold in pain-free subjects, in patients with chronic regional pain syndromes, and in patients with fibromyalgia syndrome. Arthritis Rheum, 36:642-6.

Guedj E, Cammilleri S, Colavolpe C, et al. 2007a. Predictive value of brain perfusion SPECT for ketamine response in hyperalgesic fibromyalgia. Eur J Nucl Med Mol Imaging, 34:1274-9.

Guedj E, Taieb D, Cammilleri S, et al. 2007b. 99mTc-ECD brain perfusion SPECT in hyperalgesic fibromyalgia. Eur J Nucl Med Mol Imaging, 34:130-4.

Harris RE, Clauw DJ, Scott DJ, et al. 2007. Decreased central mu-opioid receptor availability in fibromyalgia. $J$ Neurosci, 27:10000-6.

Harris RE, Gracely RH, McLean SA, et al. 2006. Comparison of clinical and evoked pain measures in fibromyalgia. $J$ Pain, 7:521-7.

Harris RE, Sundgren PC, Pang Y, et al. 2008. Dynamic levels of glutamate within the insula are associated with improvements in multiple pain domains in fibromyalgia. Arthritis Rheum, 58:903-7.

Heim C, Newport DJ, Bonsall R, et al. 2001. Altered pituitary-adrenal axis responses to provocative challenge tests in adult survivors of childhood abuse. Am J Psychiatry, 158:575-81.

Hudson JI, Goldenberg DL, Pope HGJ, et al. 1993. Comorbidity of fibromyalgia with medical and psychiatric disorders. Am J Med, 92:363-7.

Hudson JI, Hudson MS, Pliner LF, et al. 1985. Fibromyalgia and major affective disorder: A controlled phenomenology and family history study. Am J Psychiatry, 142:441-6.

Hyams KC, Wignall FS, Roswell R. 1996. War syndromes and their evaluation: from the US Civil War to the Persian Gulf War. Ann Intern Med, 125:398-405.

Jacobsen S, Bredkjaer SR. 1992. The prevalence of fibromyalgia and widespread chronic musculoskeletal pain in the general population. Scand J Rheumatol, 21:261-3.

Jacobsen S, Danneskiold-Samsoe B, Andersen RB. 1991. Oral S-adenosylmethionine in primary fibromyalgia. Double-blind clinical evaluation. Scand J Rheumatol, 20:294-302.

Jensen K, Andersen HO, Olesen J, et al. 1986. Pressure-pain threshold in human temporal region. Evaluation of a new pressure algometer. Pain, 25:313-23.
Julien N, Goffaux P, Arsenault P, et al. 2005. Widespread pain in fibromyalgia is related to a deficit of endogenous pain inhibition. Pain, 114:295-302.

Kato K, Sullivan PF, Evengard B, et al. 2006b. Chronic widespread pain and its comorbidities: a population-based study. Arch Intern Med, $166: 1649-54$

Kato K, Sullivan PF, Evengard B, et al. 2006a. Importance of genetic influences on chronic widespread pain. Arthritis Rheum, 54:1682-6.

Khan AA, Khan A, Harezlak J, et al. 2003. Somatic symptoms in primary care: etiology and outcome. Psychosomatics, 44:471-8.

Kop WJ, Lyden A, Berlin AA, et al. 2005. Ambulatory monitoring of physical activity and symptoms in fibromyalgia and chronic fatigue syndrome. Arthritis Rheum, 52:296-303.

Kosek E, Hansson P. 1997. Modulatory influence on somatosensory perception from vibration and heterotopic noxious conditioning stimulation (HNCS) in fibromyalgia patients and healthy subjects. Pain, 70:41-51.

Kwiatek R, Barnden L, Tedman R, et al. 2000. Regional cerebral blood flow in fibromyalgia: single-photon-emission computed tomography evidence of reduction in the pontine tegmentum and thalami. Arthritis Rheum, 43:2823-33.

Lautenbacher S, Rollman GB. 1997. Possible deficiencies of pain modulation in fibromyalgia. Clin J Pain, 13:189-96.

Leffler AS, Hansson P, Kosek E. 2002. Somatosensory perception in a remote pain-free area and function of diffuse noxious inhibitory controls (DNIC) in patients suffering from long-term trapezius myalgia. Eur $J$ Pain, 6:149-59.

Maixner W, Fillingim R, Booker D, et al. 1995. Sensitivity of patients with painful temporomandibular disorders to experimentally evoked pain. Pain, 63:341-51.

Mayer EA, Raybould HE. 1990. Role of visceral afferent mechanisms in functional bowel disorders. Gastroenterology, 99:1688-704.

McBeth J, Silman AJ, Gupta A, et al. 2007. Moderation of psychosocial risk factors through dysfunction of the hypothalamic-pituitary-adrenal stress axis in the onset of chronic widespread musculoskeletal pain: findings of a population-based prospective cohort study. Arthritis Rheum, 56:360-71.

McLean SA, Clauw DJ. 2004. Predicting chronic symptoms after an acute "stressor"- lessons learned from 3 medical conditions. Med Hypotheses, 63:653-8.

Moldofsky H, Scarisbrick P, England R, et al. 1975. Musculosketal symptoms and non-REM sleep disturbance in patients with "fibrositis syndrome" and healthy subjects. Psychosom Med, 37:341-51.

Moshiree B, Price DD, Robinson ME, et al. 2007. Thermal and visceral hypersensitivity in irritable bowel syndrome patients with and without fibromyalgia. Clin J Pain, 23:323-30.

Mountz JM, Bradley LA, Modell JG, et al. 1995. Fibromyalgia in women. Abnormalities of regional cerebral blood flow in the thalamus and the caudate nucleus are associated with low pain threshold levels. Arthritis Rheum, 38:926-38.

Naliboff BD, Derbyshire SW, Munakata J, et al. 2001. Cerebral activation in patients with irritable bowel syndrome and control subjects during rectosigmoid stimulation. Psychosom Med, 63:365-75.

Norregaard J, Volkmann H, Danneskiold-Samso, B. 1995. A randomized controlled trial of citalopram in the treatment of fibromyalgia. Pain, 61:445-9.

O’Malley PG, Balden E, Tomkins G, et al. 2000. Treatment of fibromyalgia with antidepressants. A meta-analysis. J Gen Intern Med, 15:659-66.

Offenbaecher M, Bondy B, de Jonge S, et al. 1999. Possible association of fibromyalgia with a polymorphism in the serotonin transporter gene regulatory region. Arthritis Rheum, 42:2482-8.

Peleg R, Ablin JN, Peleg A, et al. 2007. Characteristics of fibromyalgia in Muslim Bedouin women in a primary care clinic. Semin Arthritis Rheum, 37:398-402.

Petzke F, Clauw DJ, Ambrose K, et al. 2003a. Increased pain sensitivity in fibromyalgia: effects of stimulus type and mode of presentation Pain, 105:403-13. 
Petzke F, Gracely RH, Khine A, et al. 1999. Pain sensitivity in patients with fibromyalgia (fibromyalgia): Expectancy effects on pain measurements. Arthritis Rheum, 42:S342.

Petzke F, Gracely RH, Park KM, et al. 2003b. What do tender points measure? Influence of distress on 4 measures of tenderness. J Rheumatol, 30:567-74.

Petzke F, Khine A, Williams D, et al. 2001. Dolorimetry performed at 3 paired tender points highly predicts overall tenderness. J Rheumatol, 28:2568-9.

Pillow DR, Zautra AJ, Sandler I. 1996. Major life events and minor stressors: identifying mediational links in the stress process. J Pers Soc Psychol, 70:381-94.

Raphael KG, Natelson BH, Janal MN, et al. 2002. A community-based survey of fibromyalgia-like pain complaints following the World Trade Center terrorist attacks. Pain, 100:131-9.

Raspe H. 1992. [Rheumatism epidemiology in Europe]. Soz Praventivmed, 37:168-78.

Russell IJ, Vaeroy H, Javors M, et al. 1992. Cerebrospinal fluid biogenic amine metabolites in fibromyalgia/fibrositis syndrome and rheumatoid arthritis. Arthritis Rheum, 35:550-6.

Sapolsky RM. 1996. Why stress is bad for your brain. Science, 273:749-50.

Sarchielli P, Di Filippo M, Nardi K, et al. 2007. Sensitization, glutamate, and the link between migraine and fibromyalgia. Curr Pain Headache Rep, 11:343-51.

Scudds RA, McCain GA, Rollman GB, et al. 1989. Improvements in pain responsiveness in patients with fibrositis after successful treatment with amitriptyline. J Rheumatol Suppl, 19:98-103.

Smythe HA, Moldofsky H. 1977. Two contributions to understanding of the "fibrositis" syndrome. Bull Rheum Dis, 28:928-31.

Unwin C, Blatchley N, Coker W, et al. 1999. Health of UK servicemen who served in Persian Gulf War. Lancet, 353:169-78.

Vitton O, Gendreau M, Gendreau J, et al. 2004. A double-blind placebocontrolled trial of milnacipran in the treatment of fibromyalgia. Hum Psychopharmacol, 19:S27-S35.

Wessely S, Nimnuan C, Sharpe M. 1999. Functional somatic syndromes: One or many? Lancet, 354:936-9.

White KP, Thompson J. 2003. Fibromyalgia syndrome in an Amish community: a controlled study to determine disease and symptom prevalence. J Rheumatol, 30:1835-40.
Wilder-Smith CH, Robert-Yap J. 2007. Abnormal endogenous pain modulation and somatic and visceral hypersensitivity in female patients with irritable bowel syndrome. World J Gastroenterol, 13:3699-704.

Williams DA, Brown SC, Clauw DJ, et al. 2003. Self-reported symptoms before and after September 11 in patients with fibromyalgia. JAMA, 289:1637-8.

Wolfe F. 1997. The relation between tender points and fibromyalgia symptom variables: evidence that fibromyalgia is not a discrete disorder in the clinic. Ann Rheum Dis, 56:268-71.

Wolfe F, Ross K, Anderson J, et al. 1995. Aspects of fibromyalgia in the general population: Sex, pain threshold, and fibromyalgia symptoms. $J$ Rheumatol, 22:151-6.

Wolfe F, Smythe HA, Yunus MB, et al. 1990. The American College of Rheumatology 1990 Criteria for the Classification of Fibromyalgia. Report of the Multicenter Criteria Committee. Arthritis Rheum, 33:160-72.

Woolf CJ. 2004. Pain: moving from symptom control toward mechanism-specific pharmacologic management. Ann Intern Med, 140:441-51.

Yunus M, Masi AT, Calabro JJ, et al. 1981. Primary fibromyalgia (fibrositis): Clinical study of 50 patients with matched normal controls. Semin Arthritis Rheum, 11:151-71.

Yunus MB. 2008. Central sensitivity syndromes: a new paradigm and group nosology for fibromyalgia and overlapping conditions, and the related issue of disease versus illness. Semin Arthritis Rheum, 37:339-52.

Yunus MB. 1992. Towards a model of pathophysiology of fibromyalgia: Aberrant central pain mechanisms with peripheral modulation. $J$ Rheumatol, 19:846-50.

Yunus MB, Dailey JW, Aldag JC, et al. 1992. Plasma tryptophan and other amino acids in primary fibromyalgia: A controlled study. J Rheumatol, 19:90-4.

Yunus MB, Young CS, Saeed AS, et al. 2004. Positron emission tomography imaging of the brain in fibromyalgia syndrome. Arthritis Rheum, 51:513-8

Yunus MB, Young CS, Saeed AS, et al. 1997. Positron emission tomography (PET) imaging of the brain in fibromyalgia syndrome (fibromyalgias). Arthritis Rheum, 40:S188. 